\title{
Index modulation assisted DCT-OFDM with Enhanced Transceiver Design
}

\author{
Chang $\mathrm{He}^{1}$, Aijun Cao ${ }^{1}$, Lixia Xiao ${ }^{1}$, Lei Zhang ${ }^{2}$, Pei Xiao ${ }^{1}$, and Konstantinos Nikitopoulos ${ }^{1}$ \\ ${ }^{1}$ Institute for Communication Systems, University of Surrey, United Kingdom, GU2 7XH \\ ${ }^{1}\{$ c.he, aj.cao, 1l.xiao, p.xiao, k.nikitopoulos\}@surrey.ac.uk, \\ ${ }^{2}$ School of Engineering, University of Glasgow, Glasgow, G12 8QQ, UK \\ ${ }^{2}$ Lei.Zhang@glasgow.ac.uk
}

\begin{abstract}
An index modulation (IM) assisted Discrete Cosine Transform based Orthogonal Frequency Division Multiplexing (DCT-OFDM) with Enhanced Transmitter Design (termed as EDCT-OFDM-IM) is proposed. It amalgamates the concept of Discrete Cosine Transform assisted Orthogonal Frequency Division Multiplexing (DCT-OFDM) and Index Modulation (IM) to exploit the design freedom provided by the double number of available subcarrier under the same bandwidth. In the proposed EDCT-OFDM-IM scheme, the maximum likelihood (ML) detector used for symbol bits and index bits recovering is derived and the sophisticated designing guidelines for EDCTOFDM-IM are provided. Based on the derived pairwise error event probability, a theoretical upper bound on the average biterror probability (ABEP) of EDCT-OFDM-IM is provided over multipath fading channels. Furthermore, the maximum peak-toaverage power ratio (PAPR) of our proposed EDCT-OFDM-IM scheme is derived and compared to than the general Discrete Fourier Transform (DFT) based OFDM-IM counterpart.
\end{abstract}

\section{INTRODUCTION}

The orthogonal frequency division multiplexing (OFDM) technique, as an effective multicarrier modulation scheme, has been extensively studied and applied in many wireless communications scenarios, such as wireless Local Area Net (LAN), Long Term Evaluation (LTE) and LTE-Advanced (LTE-A) [1], [2]. Inspired by the spatial modulation conception, OFDM with index modulation (which is generally called OFDMIM) is firstly introduced as a modified variant of OFDM family. The key idea of OFDM-IM is that, by splitting the incoming bit stream into sub-groups consisting of index bits and constellation symbol bits, only the selected subcarriers are activated to modulate the corresponding data symbols while the rest subcarriers are idle for data transmission [3], [4], [5]. Since some subcarriers are selected to be deactivated, OFDMIM could suffer less PAPR and ICI effect compared to classical OFDM [6], [7], [8]. In addition, OFDM-IM offers appealing trade-offs between error performance, system complexity and data rate, when compared with classic OFDM systems [3], and its recently proposed variant waveforms such as filtered OFDM [9], filterbank multicarrier (FBMC) [10], universial filtered multicarrier(UFMC) [11].

However, the above-mentioned OFDM-IM scheme is generally implemented by the discrete Fourier transform (DFT) and inverse DFT (IDFT) modules. In fact, there exists another OFDM scheme using a cosinusoidal set as an orthogonal basis, and can be implemented with a discrete cosine transform
(DCT) module [12]. We terms this scheme as DCT-OFDM, while the general OFDM system is denoted as DFT-OFDM in this paper. In the literature, the DCT-OFDM has been proposed for many communication scenarios, such as Underwater Acoustic Communications (UAC) [13] and Visible Light Communications (VLC) [14], and noticeable improvement in the system performance over its DFT-OFDM counterpart are verified.

In general, the system implementation of using a DCT rather than a DFT brings many promising benefits for DCTOFDM. Particularly, for one-dimensional modulations (e.g., binary phase shift keying (BPSK)), the DCT-OFDM does not have the in-phase and quadrature-phase imbalance problem encountered in [15] in classic DFT-OFDM systems without the need of a quadrature modulator. In addition, unlike the traditional DFT-OFDM scheme, only half the minimum subcarrier spacing of typical DFT-OFDM is required for the DCTOFDM needs[16], [17], [18]. Consequently, the number of subcarriers in the DCT-OFDM system occupying the same bandwidth is doubled. In order to exploit this benefit of the increased number of subcarrier for IM assisted DCT-OFDM (termed as DCT-OFDM-IM), the pioneer DCT-OFDM-IM is implemented by Marwa in [18] and it has been investigated to achieve significant spectrum efficiency (SE) gains over the DFT-OFDM-IM counterpart. However, as the transceiver structure of the pioneer DCT-OFDM-IM is implemented by conventional method and its corresponding detector is based on linear detection approach, its bit error rate (BER) performance gain is limited. On the other hand, the research on DCT-OFDM-IM is still in its infancy and there is a lot of scope for further performance improvement.

Against the above background, the DCT-OFDM-IM with an enhanced transceiver (termed as EDCT-OFDM-IM) is implemented based on the pre-filtering method. In addition, the optimum maximum likelihood (ML) detector is implemented for EDCT-OFDM-IM to achieve the optimum system performance. Consequently, based on a simplified general error probability function which only considers the dominated error cases, a theoretical upper bound on the average bit-error probability is derived to evaluate the BER performance of our proposed EDCT-OFDM-IM. Furthermore, the maximum PAPR is characterized for our proposed EDCT-OFDM-IM systems. 
The remainder of this paper is organized as follows. Section II describes the pioneer DCT-OFDM-IM model proposed in [18], [19]. The transceiver structure of our proposed EDCTOFDM-IM is presented in Section III. To evaluate its performance of the EDCT-OFDM-IM scheme, we derive the corresponding theoretical BER analysis in Section IV. Furthermore, Section $\mathrm{V}$ gives the maximum PAPR value our proposed EDCT-OFDM-IM. In Section VI our numerical results are provided, while Section VII concludes this paper.

Notations: $E[\cdot]$ calculate the expected value of any random variable. The hermitian conjugate and transpose operation are represented by $[\cdot]^{H}$ and $[\cdot]^{T}$, respectively. The dimension $N \times$ $N$ matrix $\mathbf{I}_{N}$ and $\mathbf{J}_{N}$, are the identity matrix and reversal matrix, respectively. $[\cdot]^{*}$ stands for the conjugate conversion of the identified element. $(L, K, N)$ denotes a IM scheme where $K$ subcarriers are activated within a group of $L$ subcarriers, Where $N$ is the total number of available subcarriers.

\section{PIONEER DCT-OFDM-IM}

\section{A. Transmitter}

Firstly, a stream of the raw information bits is separated into $G$ groups, with each group containing $P$ bits. Then, the $P$ bits are classified into two parts with $P_{1}$ and $P_{2}$ bits for each, such that $P=P_{1}+P_{2}$. By searching a look-up table, $P_{1}$ bits for each group are modulated to an index symbol set on the active subcarriers. On the other hand, a real $M$-ary ASK constellation is employed for mapping $P_{2}$ bits. Following the index modulated symbols, a vector of length $N$ is created by performing IDCT procedure. Finally, after adding cyclic prefix $(\mathrm{CP})$ to avoid ISI and ICI, the output signal is ready for transmission.

\section{B. Receiver}

After removing the prefix and performing the DCT and block de-interleving, frequency domain zero forcing (ZF) equalization is performed on the received signal, while a minimum distance detection scheme is introduced to recover the raw information bits. Note that such a linear detection scheme results in suboptimal performance, consequently, there is need for an enhanced detector design to improve the system performance.

\section{PROPOSED EDCT-OFDM-IM SYSTEM MODEL}

Generally speaking, the major limitation of the DCT-OFDM scheme is that when the channel is in a multipath fading case, the circular convolution property which is always satisfied by DFT does not hold for DCT [12], [20]. In this regard, the cyclic-prefix (CP) based methods employed in general DFTOFDM schemes to tackle the ICI and ISI issues do not apply any more. To address this issue, and further improve the DCTOFDM-IM performance in [18], in this section, the optimum DCT-OFDM transceiver structure proposed in [21], [17], [19] is employed by cooperating with a time-reversed pre-filter and symmetric extended guard sequences to render the effective channel matrix diagonalizable by DCT in frequency domain, where one-tap equalization becomes applicable at the receiver side. In cooperation with this pre-filter, both prefix and suffix at no less than the channel memory are required as guard interval in the form of symmetric extension from modulated signal.

\section{A. Proposed EDCT-OFDM-IM transmitter}

Consider a EDCT-OFDM-IM system employs $N$ subcarriers, and the total subcarriers are separated into $G$ groups with each consisting of $L=N / G$ subcarriers. The schematic of the EDCT-OFDM-IM transmitter is illustrated in Fig. 2 (a). We denote each group by $\mathbf{Y}_{g}=\left[Y_{(g, 0)}, Y_{(g, 1)}, \ldots, Y_{(g, L-1)}\right]^{T}$, $g=0,1, \ldots, G-1$. Then the data symbol vector can be given as:

$$
\hat{\mathbf{x}}=\left[\mathbf{Y}_{0}^{T}, \mathbf{Y}_{1}^{T}, \ldots, \mathbf{Y}_{G-1}^{T}\right]^{T} .
$$

For each group, we activate $K$ out of $L$ available subcarriers to map $p_{1}=\left\lfloor\log _{2} C_{L}^{K}\right\rfloor$ bits into a set of subcarrier indices combinations, while the remaining indices transmit zeros. On the other hand, $p_{2}=K \log _{2}(M)$ bits are modulated to $M$ ary ASK data symbols and subsequently transmitted by the $K$ active subcarriers. Accordingly, the total number of bits transmitted per EDCT-OFDM-IM block is $G \cdot\left(p_{1}+p_{2}\right)$. After subcarrier IM, the $g$-th group signal can be written as

$\mathbf{Y}_{g}=\left[0, \ldots, s_{(g, 0)}, 0, \ldots, s_{(g, 1)}, 0, \ldots, s_{(g, K-1)}, 0, \ldots\right]^{T}$,

where $s_{(g, k)}(k=0,1, \ldots, K-1)$ represents the $M$-ary ASK constellation point, and $\mathbf{Y}_{g} \in \Lambda$, where $\Lambda$ is the set of all possible symbol vector combinations. In order to combat the correlation effect among adjacent channel coefficients to further improve its error performance, a subcarrier level block interleaving is added into the aforementioned system. As a result, the modified transmitted signals can be expressed as

$$
\begin{aligned}
\mathbf{x} & =\left[X_{0}, X_{1}, \ldots, X_{N-1}\right] \\
& =\left[Y_{(0,0)}, Y_{(1,0)}, \ldots, Y_{(G-1,0)}, \ldots, Y_{(0, L-1)}, Y_{(1, L-1)}, \ldots, Y_{(G-1, L-1)}\right] .
\end{aligned}
$$

The prefix and suffix are assumed to have the same length $v$. Therefore, the total block length is $L_{1}=N+2 v$ and the transmitted signal vector is represented by

$$
\mathbf{u}=\mathbf{T}_{P S} \mathbf{D}^{H} \mathbf{x}
$$

where $\mathbf{D} \in \mathbb{R}^{N \times N}$ is a power normalised type-II DCT matrix with its $(l, m)$ entry given by:

$$
d_{l, m}= \begin{cases}\sqrt{\frac{2}{N}} \cos \left(\frac{(l-1)(2 m-1) \pi}{2 N}\right), & l>1 \\ \sqrt{\frac{1}{N}}, & l=1 .\end{cases}
$$

$\mathbf{T}_{P S}=\left[\mathbf{J}_{v}, \mathbf{0}_{v \times(N-v)} ; \mathbf{I}_{N} ; \mathbf{0}_{v \times(N-v)}, \mathbf{J}_{v}\right]$ is the $L_{1} \times N$ matrix that inserts the prefix and suffix at both sides of a data symbol block.

\section{B. Proposed EDCT-OFDM-IM receiver}

Fig. 2 (b) depicts the receiver structure of the proposed EDCT-OFDM-IM system. Assume a Rayleigh fading channel vector at $L_{c}$ taps in the time domain as defined by $\mathbf{h}=\left[h_{0}, h_{1}, \ldots, h_{L_{c}-1}\right]$. Its corresponding time-reverse prefilter vector is thus represented in the reverse form as $\mathbf{p}=$ $\left[h_{L_{c}-1}, h_{L_{c}-2}, \ldots, h_{0}\right]$, 


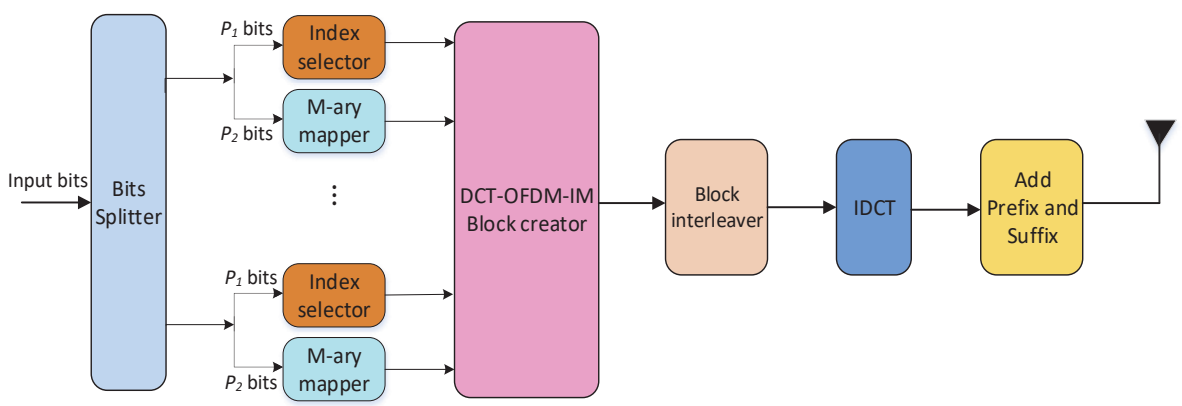

(a)

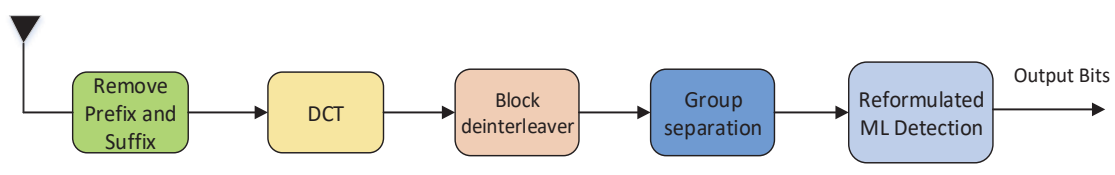

(b)

Fig. 1. Transceiver diagram of the proposed EDCT-OFDM-IM scheme (a) transmitter side (b) receiver side.

At the receiver side, by performing the time-reverse prefiltering and the DCT demultiplexing, the received frequencydomain sample vector obtained after guard sequence removal can be expressed as

$$
\mathbf{z}=\mathbf{D R}_{P S} \mathbf{P}(\mathbf{H u}+\mathbf{n}),
$$

where the multipath channel convolution matrix $\mathbf{H} \in \mathbb{R}^{L_{1} \times L_{1}}$ is a Toeplitz matrix defined by

$$
\mathbf{H}=\left[\begin{array}{ccccc}
h_{L_{c}-1} & \ldots & h_{0} & \ldots & 0 \\
0 & \ddots & & \ddots & \vdots \\
\vdots & \ddots & \ddots & & h_{0} \\
\vdots & & \ddots & \ddots & \vdots \\
0 & \ldots & \ldots & 0 & h_{L_{c}-1}
\end{array}\right]
$$

Correspondingly, the time-reverse filtering matrix $\mathbf{P} \in \mathbb{R}^{L_{1} \times L_{1}}$ can also be represented by a Toeplitz matrix as

$$
\mathbf{P}=\left[\begin{array}{ccccc}
h_{L_{c}-1} & 0 & \ldots & \ldots & 0 \\
\vdots & \ddots & \ddots & & \vdots \\
h_{0} & & \ddots & \ddots & \vdots \\
\vdots & \ddots & & \ddots & 0 \\
0 & \ldots & h_{0} & \ldots & h_{L_{c}-1}
\end{array}\right]
$$

The guard sequence removal operation is denoted by the matrix $\mathbf{R}_{P S}=\left[\mathbf{0}_{N \times v}, \mathbf{I}_{N}, \mathbf{0}_{N \times v}\right] . \mathbf{n} \in \mathbb{R}^{L_{1} \times 1}$ is the AWGN noise vector with its elements subject to Gaussian distribution with zero mean and $N_{0}$ variance.

Recalling the aforementioned one-tap equalization mechanism, with the introduction of a pre-filter and the symmetric guard sequence, the DCT-OFDM is now applicable for ICI and ISI free transmission. This integrates the output of
$\mathbf{D R}_{P S} \mathbf{P H T}_{P S} \mathbf{D}^{H}$ equivalent to a effective diagonal matrix $\mathbf{H}_{e f f}$ and Eq. (6) thus can be simplified to

$$
\mathbf{z}=\mathbf{H}_{e f f} \mathbf{x}+\mathbf{G}_{e f f} \mathbf{n}
$$

where $\mathbf{H}_{\text {eff }} \in \mathbb{R}^{N \times N}$ is the effective channel diagonal matrix with its elements expressed by $\operatorname{diag}\left(\mathbf{H}_{\text {eff }}\right)=$ $\left[H_{0}, H_{1}, \ldots, H_{N-1}\right]$, and $\mathbf{G}_{e f f}=\mathbf{D R}_{P S} \mathbf{P}$ represents the effective noise correlation matrix.

In conventional DFT-OFDM systems, it is suggested that the noise vector in frequency-domain after DFT process is still Gaussian variable with equal power distributed among all subcarriers. However, in this DCT-OFDM system, due to the correlated pre-filtering procedure, it becomes variant and correlated. Consequently, the ML detector which is normally used to optimally detect the transmit signal need to be reformulated by taking the noise correlation effect into account. In order to calculate the effective noise variance for each subcarrier, we take a scaled form from Eq. (9), as expressed by

$$
Z_{k}=H_{k} X_{k}+\sum_{i=1}^{N} g_{k, i} n_{i}
$$

where $Z_{k}$ and $n_{i}$ are the received sample and AWGN sample at the $k$ th subcarrier and $i$ th subcarrier, respectively, whereas $g_{k, i}$ is the entry in the $k$ th row and $i$ th column of $\mathbf{G}_{e f f}$.

We make a reasonable assumption that time is long over symbols. With this assumption, $g_{k, i}^{2}$ is regarded as instant noise coefficient and the overall coloured noise coefficient on arbitrary subcarrier index is in the summation of the noise coefficient from all subcarriers. Accordingly, the coloured noise variance $V_{k}$ at subcarrier index $k$ can be obtained as

$$
V_{k}=\frac{N_{0}}{2} \sum_{i=1}^{N} g_{k, i}^{2} \text {. }
$$


Based on the instantaneous coloured noise variance $V_{k}$, we reformulated the expression of the optimum ML detection for $g$ th group. Denoting by $Z_{g+\lambda G}=Z_{(g, \lambda)}, H_{g+\lambda G}=H_{(g, \lambda)}$ and $V_{g+\lambda G}=V_{(g, \lambda)}$, the transmit symbols in the $g$ th group can be estimated as

$$
\hat{\mathbf{Y}}_{g}=\underset{\mathbf{Y}_{g} \in \Lambda}{\arg \min } \sum_{\lambda=0}^{L-1} \frac{\left|Z_{(g, \lambda)}-H_{(g, \lambda)} Y_{(g, \lambda)}\right|^{2}}{V_{(g, \lambda)}} .
$$

As indicated in Eq. (12), the noise correlation effect can be effectively compensated by the term $V_{g+\lambda G}$.

\section{Theoretical Error Performance Analysis of THE PROPOSED EDCT-OFDM-IM}

In this section, we propose an analytical framework to evaluate the average bit error probability (ABEP) of our EDCTOFDM-IM scheme using the aforementioned reformulated ML decoder. Unlike conventional OFDM systems, the BER performance for the DCT-OFDM-IM schemes is determined by pair wise error probability (PEP) events within a divided group rather than a single subcarrier. For the EDCT-OFDMIM scheme, let $\left(\mathbf{Y}_{g} \rightarrow \tilde{\mathbf{Y}}_{g}, \mathbf{H}_{g}\right)$ denotes the pair wise error event where the transmitted signal $\mathbf{Y}_{g}$ in the $g$-th group is erroneously detected as $\tilde{\mathbf{Y}}_{g}$ conditioned on the corresponding effective channel coefficient vector $\mathbf{H}_{g}$. In this regard, the conditional PEP (CPEP) expression in [22], can be reformulated by compensating the coloured noise variance $V_{(g, \lambda)}$ on each subcarrier, and is expressed as

$$
P\left(\mathbf{Y}_{g} \rightarrow \tilde{\mathbf{Y}}_{g}, \mathbf{H}_{g}\right)=Q(\sqrt{\delta})
$$

where

$$
\delta=\sum_{\lambda=0}^{L-1} \frac{\left|H_{(g, \lambda)} Y_{(g, \lambda)}-H_{(g, \lambda)} \tilde{Y}_{(g, \lambda)}\right|^{2}}{2 V_{(g, \lambda)}}
$$

and

$$
Q(x) \approx \frac{1}{12} e^{-x^{2} / 2}+\frac{1}{4} e^{-2 x^{2} / 3}
$$

is the error function. The unconditional PEP (UPEP), is thus obtained by taking the average of the effective channel coefficients of the CPEP as

$$
P\left(\mathbf{Y}_{g} \rightarrow \tilde{\mathbf{Y}}_{g}\right)=\int_{\mathbf{H}_{g}} P\left(\mathbf{Y}_{g} \rightarrow \tilde{\mathbf{Y}}_{g}, \mathbf{H}_{g}\right) \cdot f\left(\mathbf{H}_{g}\right) d \mathbf{H}_{g}
$$

where $f\left(\mathbf{H}_{g}\right)$ is the probability density function (PDF) of the effective channel coefficient $\mathbf{H}_{g}$. However, the CPEPs of EDCT-OFDM-IM is complicated as $\mathbf{H}_{g}$ is the convolution output between the channel and its corresponding pre-filter. Instead of deriving a closed-form expression for Eq. (26), we follow [23], [24], [19] and estimate the UPEPs of Eq. (26) by numerical integration.

Next, we can define the ABEP of the proposed EDCTOFDM-IM scheme as the ratio of the number of the error bits $m_{e}$ to the number of the total transmission bits $m_{t}$, which is given as

$$
P_{b}=\frac{m_{e}}{m_{t}}
$$

In accordance with the aforementioned definitions for each group, we have $m_{t}=G \cdot\left(p_{1}+p_{2}\right)$ and $m_{e}$ is the sum of error bits among the $G$ groups, i.e., $m_{e}=\sum_{g=0}^{G-1} m_{e, g}$.

In general, $m_{e, g}$ is obtained by searching all possible PEPs within the $g$-th group exhaustively. Nevertheless, operating an exhaustive search is unnecessary and becomes impractical when the number of all possible PEPs is very large. Since some low-probability error events are safely to be ignored, we focus mainly on two dominated error cases where bit errors are most likely to occur according to [23], [24]:

(i) $\mathbf{Y}_{g}$ and $\tilde{\mathbf{Y}}_{g}$ have only one different index of activated subcarrier and the $M$-ary ASK symbols on the incorrect index can be randomly right or wrong;

(ii) $\mathbf{Y}_{g}$ and $\tilde{\mathbf{Y}}_{g}$ have correct index set of activated subcarrier but one incorrect carried $M$-ary ASK constellation symbol.

The bit errors caused by case (i) and case (ii) are denoted by $m_{e, g}^{(\mathrm{i})}$ and $m_{e, g}^{(\mathrm{ii})}$, respectively. And the total errors $m_{e, g}=$ $m_{e, g}^{(\mathrm{i})}+m_{e, g}^{(\mathrm{ii})}$.

For case (i), suppose $\alpha$ is the subcarrier index in $\mathbf{Y}_{g}$ that is incorrectly detected as $\tilde{\alpha}$ in $\tilde{\mathbf{Y}}_{g}$ where $\alpha, \tilde{\alpha} \in\{1,2, \ldots, L-1\}$ and $\alpha \neq \tilde{\alpha}$, and the corresponding ASK symbols carried by the $\alpha$-th subcarrier in $\mathbf{Y}_{g}$ and $\tilde{\alpha}$-th subcarrier in $\tilde{\mathbf{Y}}_{g}$ are $Y_{(g, \alpha)}$ and $\tilde{Y}_{(g, \tilde{\alpha})}$, respectively. Then, the CPEP given in Eq. (16) which indicates the error event of the activated subcarrier $\alpha$ being erroneously detected as $\tilde{\alpha}$ can be simplified to

$P\left(\mathbf{Y}_{g} \rightarrow \tilde{\mathbf{Y}}_{g} \mid \alpha \rightarrow \tilde{\alpha}, \mathbf{H}_{g}\right)=Q\left(\sqrt{\frac{\left|H_{(g, \alpha)} Y_{(g, \alpha)}\right|^{2}}{2 V_{(g, \alpha)}}+\frac{\left|H_{(g, \tilde{\alpha})} \tilde{Y}_{(g, \tilde{\alpha})}\right|^{2}}{2 V_{(g, \tilde{\alpha})}}}\right)$.

Subsequently, $m_{e, g}^{(\mathrm{i})}$ of EDCT-OFDM-IM for case (i) is obtained by averaging $P\left(\mathbf{Y}_{g} \rightarrow \tilde{\mathbf{Y}}_{g} \mid \alpha \rightarrow \tilde{\alpha}, \mathbf{H}_{g}\right)$ over all possible events of $(\alpha \rightarrow \tilde{\alpha})$ as

$m_{e, g}^{(\mathbf{i})}=\frac{1}{L} \sum_{\alpha=0}^{L-1} \sum_{\tilde{\alpha}=0, \tilde{\alpha} \neq \alpha}^{L-1} P\left(\mathbf{Y}_{g} \rightarrow \tilde{\mathbf{Y}}_{g} \mid \alpha \rightarrow \tilde{\alpha}\right) \cdot\left[A\left(\mathbf{Y}_{g} \rightarrow \tilde{\mathbf{Y}}_{g} \mid \alpha \rightarrow \tilde{\alpha}\right)+\frac{\log _{2} M}{2}\right]$

where $A\left(\mathbf{Y}_{g} \rightarrow \tilde{\mathbf{Y}}_{g} \mid \alpha \rightarrow \tilde{\alpha}\right)$ is the Hamming distance between $\mathbf{Y}_{g}$ and $\tilde{\mathbf{Y}}_{g}$ conditioned on $\alpha \rightarrow \tilde{\alpha}$.

For case (ii), we assume $Y_{(g, \varphi)}$ is erroneously detected as $\tilde{Y}_{(g, \varphi)}$ on the index $\varphi$ while other subcarriers recover the correct symbols. In this case, the CPEP can be a simplified as

$P\left(\mathbf{Y}_{g} \rightarrow \tilde{\mathbf{Y}}_{g} \mid Y_{(g, \varphi)} \rightarrow \tilde{Y}_{(g, \varphi)}, \mathbf{H}_{g}\right)=Q\left(\sqrt{\frac{\left|H_{(g, \varphi)} Y_{(g, \varphi)}-H_{(g, \varphi)} \tilde{Y}_{(g, \varphi)}\right|^{2}}{2 V_{(g, \varphi)}}}\right)$.

In the same manner, the $m_{e, g}^{(i i)}$ of EDCT-OFDM-IM for case (ii) is achieved by searching all possible cases of $\left(Y_{(g, \varphi)} \rightarrow\right.$ $\left.\tilde{Y}_{(g, \varphi)}\right)$, which can be expressed as

$m_{e, g}^{(\mathrm{ii})}=\frac{1}{L} \sum_{\varphi=0}^{L-1} \sum_{Y_{(g, \varphi)} \neq \tilde{Y}_{(g, \varphi)}} P\left(\mathbf{Y}_{g} \rightarrow \tilde{\mathbf{Y}}_{g} \mid Y_{(g, \varphi)} \rightarrow \tilde{Y}_{(g, \varphi)}\right) \cdot B(M)$

where $M$ is the ASK modulated constellation symbol order, and $B(M)$ represents 1 bit in error for BPSK case $(M=2)$ and half detection accuracy of $\log _{2} M$ transmit bits for $M \geq 4$ as follow

$$
B(M)=\left\{\begin{array}{lc}
1, & B P S K(M=2) \\
\frac{\log _{2} M}{2}, & M-A S K(M \geq 4)
\end{array} .\right.
$$

Finally, by substituting Eq. (19) and Eq. (21) into Eq. (17), the generalized expression for the ABEP of EDCT-OFDM-IM 
system can be upper bounded as

$$
\begin{gathered}
P_{b}=\frac{m_{e}}{G \cdot\left(p_{1}+p_{2}\right)} \leq \frac{1}{G \cdot\left(p_{1}+p_{2}\right)} \sum_{g=0}^{G-1}\left[m_{e, g}^{(\mathrm{i})}+m_{e, g}^{(\mathrm{ii})}\right] . \\
\text { V. The MAXIMUM VALUE OF PAPR }
\end{gathered}
$$

\section{The Maximum Value of PAPR}

The maximum signal power in DFT-based OFDM system is normally derived by assuming that each subcarrier is carried with highest symbol power and all the complex exponentials are in phase to each other. This yields the highest aggregation power as a summation of several constellation symbols. However, in the DCT-based OFDM systems, since the transmitted signal is attenuated by the cosine terms from IDCT rather than the complex exponential terms from IDFT, we start the derivation from the expression of the modulated signal ready to be transmitted.

After the IDCT modulation procedure, the time domain symbols to be transmitted in the DCT-based OFDM system is expressed as:

$$
s_{k}=\sum_{n=0}^{N-1} X_{n} \beta_{n} \cos \left[\frac{\pi n(2 k+1)}{2 N}\right], \quad 0 \leq k \leq N-1 .
$$

In general, the PAPR is defined as the ratio of the maximum achievable signal power, $P_{\max }$, and the average signal power, $P_{\text {mean }}$ :

$$
P A P R=\frac{P_{\text {max }}}{P_{\text {mean }}}=\frac{\max \left\{\left|s_{k}\right|^{2}\right\}}{E\left\{\left|s_{k}\right|^{2}\right\}} .
$$

As indicated by Eq. (25), the peak power of $s_{k}$ occurs when $\cos \left[\frac{\pi n(2 k+1)}{2 N}\right]= \pm 1$, which is achieved at $k=N-1$. Accordingly, the absolute amplitude of the term $X_{n} \beta_{n} \cos \left[\frac{\pi n(2 k+1)}{2 N}\right]$ becomes maximum with its polarization either being positive or negative. Hence, the maximum possible power is achieved at $s_{N-1}$ when each subcarrier is modulated with the peak of constellation symbol amplitude and identical polarization with the cosine term. Suppose the greatest constellation symbol amplitude of $X_{n}$ in $M$-ary ASK modulation scheme is denoted as $\max \left|X_{n}\right|=X_{\max }$, it satisfies $X_{\max }=M-1$. For the conventional DCT-based OFDM system where all subcarriers are modulated with symbols, the maximum possible signal power is

$$
P_{\max } \approx \max \left|\sum_{n=0}^{N-1} X_{\max } \beta_{n}\right|^{2} \approx N(M-1)^{2} .
$$

On the other hand, $P_{\text {mean }}$ depends on the average energy distributed on each subcarrier multiplied by the number of modulated subcarriers. The average energy per subcarrier is the same to that in an $M$-ary ASK modulated symbol. In this regard, we have

$$
P_{\text {mean }}=\frac{E_{\text {mean }}^{\text {ask } N}}{N}=\frac{M^{2}-1}{3} .
$$

By combining Eq. (26) and Eq.(27), the PAPR for the conventional DCT-based OFDM system can be calculated as:

$$
P A P R_{D C T}=\frac{P_{\text {max }}}{P_{\text {mean }}}=\frac{3 N(M-1)}{M+1} .
$$

However, for DCT-OFDM-IM systems, the fact that some subcarriers are deactivated due to the index modulation suggests a power reduction on both $P_{\max }$ and $P_{\text {mean }}$. As partial subcarriers are set to transmit zeros, the maximum possible signal power and the average signal power are proportional to the total number of active subcarriers. With the aforementioned assumptions, for a general DCT-OFDM-IM $(L, K, N) M$ ASK scheme, the total active subcarrier number is $N_{a}=$ $N K / L$. Hence, $P_{\max }$ and $P_{\text {mean }}$ are calculated by

$$
\begin{gathered}
P_{\text {max }} \approx\left|\frac{1}{\sqrt{N}} \cdot \frac{N K}{L} X_{\text {max }}\right|^{2}=\frac{N K^{2}(M-1)^{2}}{L^{2}} \\
P_{\text {mean }}=\frac{E_{\text {mean }}^{\text {ask }} \cdot N K / L}{N}=\frac{K\left(M^{2}-1\right)}{3 L} .
\end{gathered}
$$

Consequently, we have the PAPR for general DCT-OFDM-IM:

$$
P A P R_{D C T}^{I M}=\frac{3 N K(M-1)}{L(M+1)}=\frac{3 N_{a}(M-1)}{(M+1)} .
$$

The expression in Eq. (31) reveals the advantage of the general DCT-OFDM-IM, i.e., just like other kind of IM-based variants where $N_{a}<N$, the PAPR is subject to the number of total active subcarriers and could be reduced if less subcarriers are modulated for carrying symbols. By contrast, the worst PAPR is obtained when $N_{a}=N$.

Furthermore, the PAPR of the conventional DFTbased OFDM and DFT-OFDM-IM schemes are given by $P A P R_{D F T}=\frac{3 \mathcal{N}(\sqrt{\mathcal{M}}-1)}{\sqrt{\mathcal{M}}+1}$ and PAPR $R_{D F T}^{I M}=\frac{3 \mathcal{N} \mathcal{K}(\sqrt{\mathcal{M}}-1)}{\mathcal{L}(\sqrt{\mathcal{M}}+1)}$, respectively [7]. For conventional DFT-based $\mathcal{M}$-QAM modulated and DCT-based $M$-ASK modulated OFDM systems with the same spectral efficiency (e.g. $N=2 \mathcal{N}$, $\mathcal{M}=M^{2}$ ), it is easy to obtain $P A P R_{D C T}=2 P A P R_{D F T}$. Due to the double number of available subcarrier, the DCTbased scheme suffers from a higher PAPR in comparison with the conventional OFDM systems. Nevertheless, when it comes to IM-based cases, in accordance with the grouping principles we proposed in the spectral efficiency analysis, their total active subcarrier number in a frame are the same. Hence, the general DCT-OFDM-IM schemes show similar PAPR effect as the DFT-OFDM-IM schemes, which is $P A P R_{D C T}^{I M}=P A P R_{D F T}^{I M}$.

\section{NUMERICAL RESULTS}

In this section, we present our simulation results for characterizing the achievable performance of our proposed EDCTOFDM-IM system. For fair comparisons, the normalised transmission rate and the transmit power are set to the identical for all the schemes.

We consider a ten-path (i.e. $L_{c}=10$ ) slow-varying Rayleigh fading channel with exponential power delay profile, and the channel information is supposed to be perfectly obtained by the receiver. Moreover, we assign the DFT-based systems with $\mathcal{N}=64$ subcarriers and a cyclic prefix of length of 16 whereas the DCT-based systems are assigned with $N=128$ subcarriers and a symmetric prefix and suffix of the same length of $v=$ 16. 


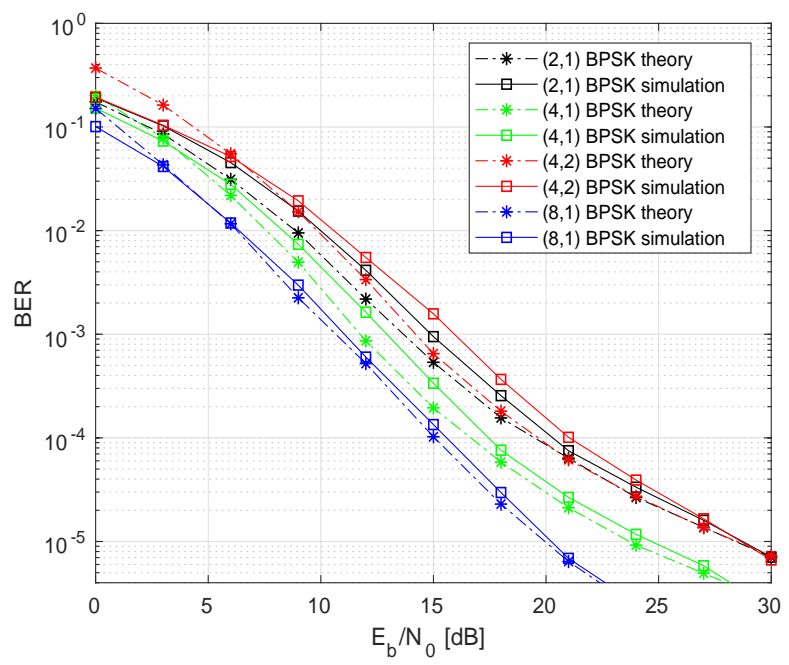

Fig. 2. Performance of EDCT-OFDM-IM for different index modulation setups.

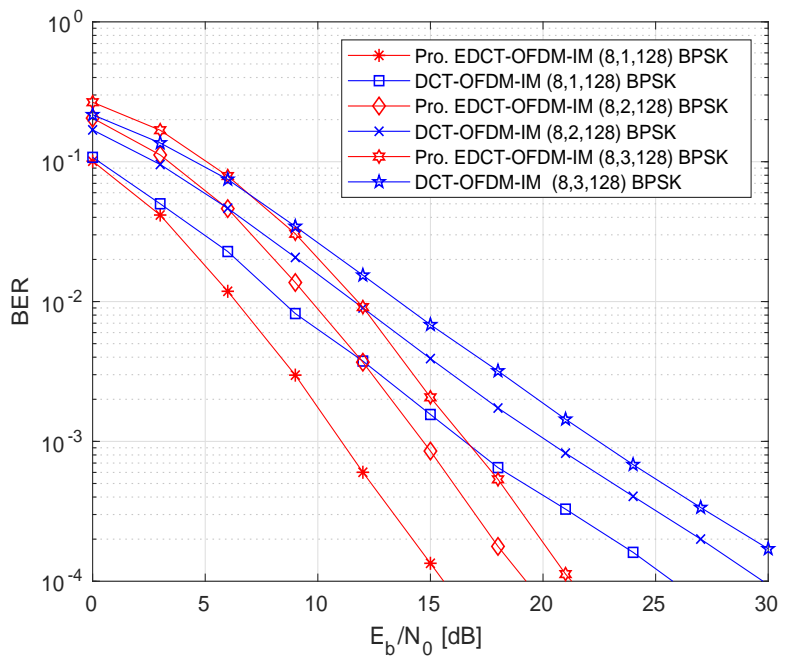

Fig. 3. Performance comparison for the proposed EDCT-OFDM-IM and DCT-OFDM-IM.

First, to verify the tightness of the theoretical BER upper bound presented in Eq. (23), we compare the analytical and simulation results with several different system configurations in Fig. 2. In all the cases, the curves for theoretical BER upper bound are close to their corresponding simulation results with less than $1 \mathrm{~dB}$ gap in between. The accuracy improves further when the average BERs are lower than $10^{-3}$. On the other hand, the figure also indicates that the accuracy of our theoretical analysis improves as the group size $L$ increases.

Next, we show the BER performance comparison for our proposed EDCT-OFDM-IM and the pioneer DCT-OFDM-IM with the same setups in Fig. 3. It can be seen that with the optimal ML detector employed instead of a linear detector, the overall system performance could be significantly improved.

The exact BER performance comparison for the classic OFDM, DFT-OFDM-IM and EDCT-OFDM-IM systems are provided in Figs. 4-5 for a normalised transmission rate at

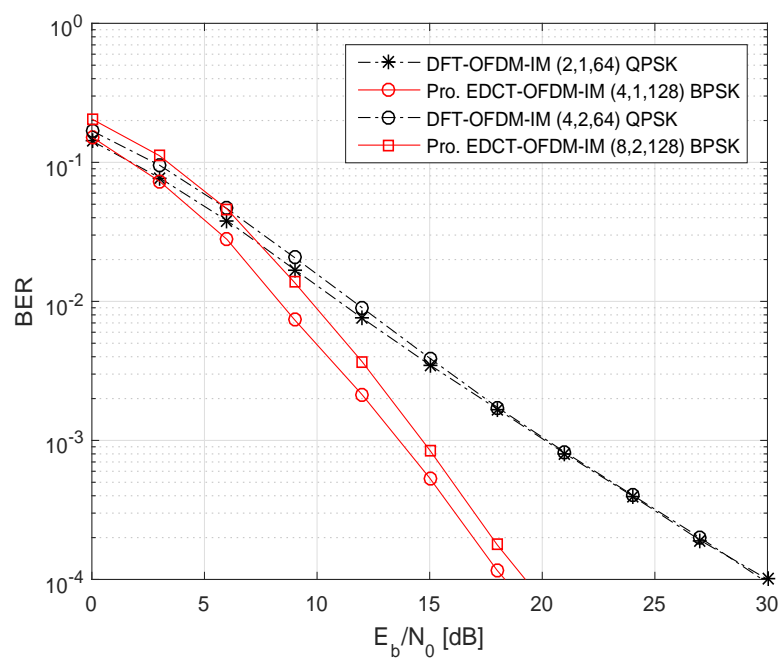

Fig. 4. Performance comparison for DFT-OFDM-IM and EDCT-OFDM-IM at $1.5 \mathrm{bits} / \mathrm{s} / \mathrm{Hz}$

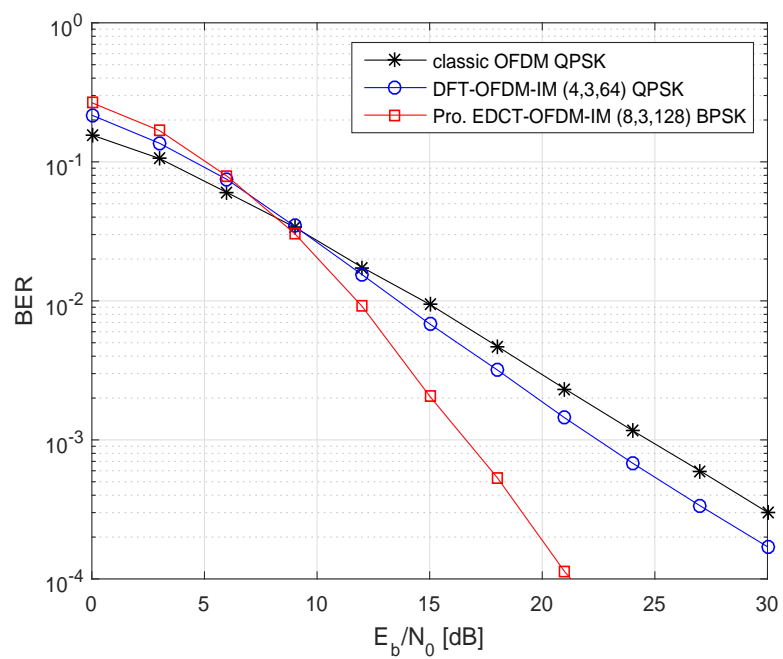

Fig. 5. Performance comparison for classic OFDM, DFT-OFDM-IM and EDCT-OFDM-IM at $2 \mathrm{bits} / \mathrm{s} / \mathrm{Hz}$.

$1.5 \mathrm{bits} / \mathrm{s} / \mathrm{Hz}$ and $2 \mathrm{bits} / \mathrm{s} / \mathrm{Hz}$, respectively. It is noted that the setups made for the proposed EDCT-OFDM-IM and the DFTOFDM-IM ensure similar detection complexity under the same date rate. It can be observed from the figures that the EDCTOFDM-IM significantly outperforms both the classic OFDM and the DFT-OFDM-IM. Specifically, The performance gain of the systems corresponding to the transmission data rate of $1.5 \mathrm{bits} / \mathrm{s} / \mathrm{Hz}$ in Fig. 4 is higher than that of the systems corresponding to the transmission data rate of $2 \mathrm{bits} / \mathrm{s} / \mathrm{Hz}$ in Fig. 5. For instance, at a BER of $10^{-3}$, around $5 \mathrm{~dB}$ gain and $6 \mathrm{~dB}$ gain are observed both in Fig. 4 and Fig. 5 for our EDCT-OFDM-IM systems.

Another reason for this observed trend can be inferred from the conclusion from some previous studies [7], [25]. In the high SNR region, the information bits carried by the index set are more reliable than the bits carried by constellation symbols, which concurs with our results provided in Fig. 6 


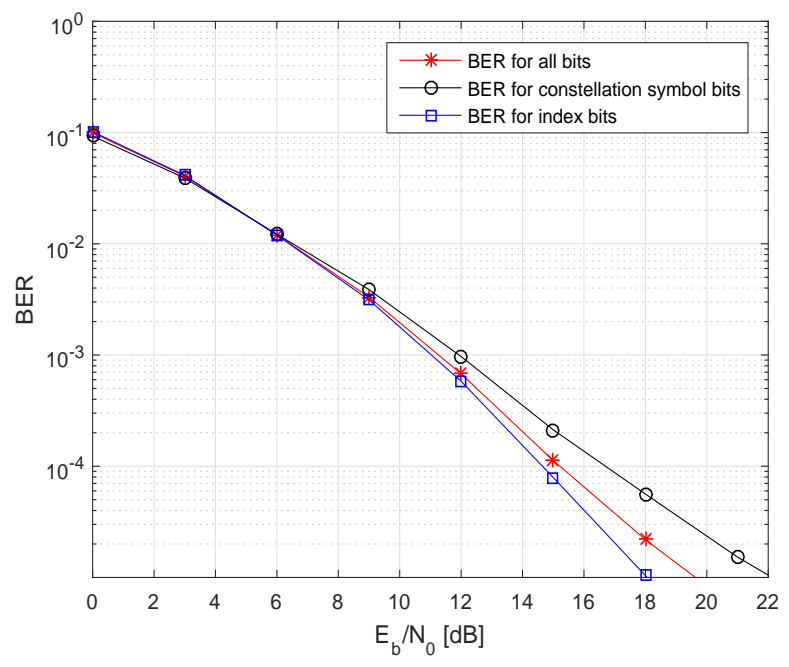

Fig. 6. BER performance on the index bits and the symbol bits for the EDCT-OFDM-IM $(8,1,128)$ BPSK scheme.

where we select the EDCT-OFDM-IM $(8,1,128)$ BPSK scheme as an example to provide the BER comparison for the index bits, the constellation symbol bits and all bits cases. The gap between index bits and symbols bits becomes larger as BER decreases. In this regard, our designing guidelines verifies the superiority of our proposed EDCT-OFDM-IM system which could reduce the number of information bits modulated by constellation symbols, but make up this data rate loss from increasing the number of index bits with the double number of available subcarriers.

\section{CONCLUSION}

In this paper, we have illustrated an enhanced transceiver design for the proposed EDCT-OFDM-IM system and carried out its comparisons with the DFT-OFDM-IM system. By exploiting the increased sparsity of the modulated symbol vector, the EDCT-OFDM-IM scheme shows an explicit BER advantage than its DFT-based counterpart under the same spectral efficiency. Moreover, we show that the PAPRs of the EDCT-OFDM-IM and the DFT-OFDM-IM systems are almost identical.

\section{ACKNOWLEDGEMENT}

This work was supported in part by the U.K. Engineering and Physical Sciences Research Council under Grant EP/R001588/1 and EP/S02476X/1. The authors also would like to acknowledge the support of the University of the Surrey 5GIC.

\section{REFERENCES}

[1] S. Weinstein and P. Ebert, "Data transmission by frequency-division multiplexing using the discrete fourier transform", IEEE Transactions on Communication Technology, vol. 19, no. 5, pp. 628-634, October 1971.

[2] B. Hirosaki, "An orthogonally multiplexed QAM system using the discrete fourier transform", IEEE Transactions on Communications, vol. 29, no. 7, pp. 982-989, July 1981.
[3] E. Basar, M. Wen, R. Mesleh, et al., "Index modulation techniques for next-generation wireless networks", IEEE Access, vol. 5, pp. 16693 16746, 2017.

[4] Y. Xiao, S. Wang, L. Dan, et al., "OFDM with interleaved subcarrierindex modulation", IEEE Communications Letters, vol. 18, no. 8, pp. 1447-1450, Aug 2014.

[5] N. Ishikawa, S. Sugiura, and L. Hanzo, "Subcarrier-index modulation aided ofdm - will it work?", IEEE Access, vol. 4, pp. 2580-2593, 2016.

[6] Lixia Xiao, Bin Xu, Huirong Bai, et al., "Performance evaluation in PAPR and ICI for ISIM-OFDM systems", in 2014 International Workshop on High Mobility Wireless Communications, Nov 2014, pp. 84-88.

[7] D. Tsonev, S. Sinanovic, and H. Haas, "Enhanced subcarrier index modulation (SIM) OFDM", in 2011 IEEE GLOBECOM Workshops (GC Wkshps), Dec 2011, pp. 728-732.

[8] T. A. Weiss and F. K. Jondral, "Spectrum pooling: an innovative strategy for the enhancement of spectrum efficiency", IEEE Communications Magazine, vol. 42, no. 3, pp. S8-14, Mar 2004.

[9] L. Zhang, A. Ijaz, P. Xiao, M. M. Molu, and R. Tafazolli, "Filtered OFDM systems, algorithms, and performance analysis for $5 \mathrm{G}$ and beyond", IEEE Transactions on Communications, vol. 66, no. 3, pp. 1205-1218, March 2018.

[10] L. Zhang, P. Xiao, A. Zafar, et al., "FBMC system: An insight into doubly dispersive channel impact", IEEE Transactions on Vehicular Technology, vol. 66, no. 5, pp. 3942-3956, May 2017.

[11] L. Zhang, A. Ijaz, P. Xiao, A. Quddus, and R. Tafazolli, "Subband filtered multi-carrier systems for multi-service wireless communications", IEEE Transactions on Wireless Communications, vol. 16, no. 3, pp. 1893-1907, March 2017.

[12] G. D. Mandyam, "Sinusoidal transforms in OFDM systems", IEEE Transactions on Broadcasting, vol. 50, no. 2, pp. 172-184, June 2004.

[13] P. Kumar and P. Kumar, "DCT based OFDM for underwater acoustic communication", in 2012 1st International Conference on Recent Advances in Information Technology (RAIT), March 2012, pp. 170-176.

[14] O. Narmanloglu and M. Uysal, "DCT-OFDM based visible light communications", in 2016 24th Signal Processing and Communication Application Conference (SIU), May 2016, pp. 521-524.

[15] A. Schuchert, R. Hasholzner, et al., "A novel IQ imbalance compensation scheme for the reception of ofdm signals", IEEE Transactions on Consumer Electronics, vol. 47, no. 3, pp. 313-318, Aug 2001.

[16] Chang He, L. Zhang, J. Mao, et al., "Output SNR analysis and detection criteria for optimum DCT-based multicarrier system", in 2016 International Symposium on Wireless Communication Systems (ISWCS), Sept 2016, pp. 59-64.

[17] C. He, L. Zhang, J. Mao, et al., "Performance analysis and optimization of DCT-based multicarrier system on frequency-selective fading channels", IEEE Access, vol. 6, pp. 13075-13089, 2018.

[18] M. Chafii, J. P. Coon, and D. A. Hedges, "DCT-OFDM with index modulation", IEEE Communications Letters, vol. 21, no. 7, pp. 14891492, July 2017.

[19] C. He, A. Cao, L. Xiao, L. Zhang, P. Xiao, and K. Nikitopoulos, "Enhanced DCT-OFDM system with index modulation”, IEEE Transactions on Vehicular Technology, 2019.

[20] V. Sanchez, P. Garcia, A. M. Peinado, et al., "Diagonalizing properties of the discrete cosine transforms", IEEE Transactions on Signal Processing, pp. 2631-2641, Nov 1995.

[21] N. Al-Dhahir et al., "Optimum DCT-based multicarrier transceivers for frequency-selective channels", IEEE Transactions on Communications, vol. 54, no. 4, pp. 760-760, April 2006.

[22] E. Basar, U. Aygolu, E. Panayrc, et al., "Orthogonal frequency division multiplexing with index modulation", IEEE Transactions on Signal Processing, vol. 61, no. 22, pp. 5536-5549, Nov 2013.

[23] Y. Ko, "A tight upper bound on bit error rate of joint ofdm and multicarrier index keying", IEEE Communications Letters, vol. 18, no. 10 , pp. 1763-1766, Oct 2014.

[24] Q. Ma, P. Yang, Y. Xiao, et al., "Error probability analysis of ofdm-im with carrier frequency offset", IEEE Communications Letters, vol. 20, no. 12, pp. 2434-2437, Dec 2016.

[25] M. Wen, B. Ye, E. Basar, et al., "Enhanced orthogonal frequency division multiplexing with index modulation", IEEE Transactions on Wireless Communications, vol. 16, no. 7, pp. 4786-4801, July 2017. 